\title{
PROYECCIONES
}

Revista de Matemática vol. 8/9 Pág. 47-63

$1989 / 90$ - ISSN 0716-0917

Universidad Católica del Norte

Antofagasta - Chile

\section{A MESH INDEPENDENCE PRINCIPLE FOR NONLINEAR EQUATIONS USING NEWTON'S METHOD AND NONLINEAR PROJECTIONS.}

\author{
Ioamnis K. Angynos
}

\author{
A B S T R A C T
}

\begin{abstract}
We consider the nonlinear operator equation in a Banach space. We make use of nonizinear projections on finite dimensional spaces to produce the finite dimensional discretization of the nonlinear equation. Using Newton's method we then prove the mesh-independence principle for this problem. Our results cover and extend previous results involving linear projections on finite dimensional spaces.
\end{abstract}

\section{INTRODUCTION}

In this paper we extend the validity of the mesh independence principle for discretizations of operators. We consider the equation

$$
F(z)=0
$$

where $F$ is a nonl inear operator on a Banach space $X$. Newton's iteration for (1) is given by

$$
z_{n+1}=z_{n}-F^{\prime}\left(z_{n}\right)^{-1} F\left(z_{n}\right)
$$

The above iteration, under certain assumptions, converges quadratically to 
a solution $z^{*}$ of (1). However, it is not at all easy in general to compute the iterates in (2). That is why we consider the discretized family of equations

$$
T_{h}(x)=0
$$

indexed by some $h>0$ to solve (1), where $T_{h}$ is a nonlinear operator on a finite dimensional space $x_{n}$. Under certain assumptions we show that the equations (3) have solutions

$$
x_{h}^{\star}=P_{h}\left(z^{\star}\right)+O\left(h^{p}\right)
$$

which are the limit of the Newton sequence applied to (3) such that:

$$
x_{0}^{h}=P_{h}\left(z_{0}\right), x_{n+1}^{h}=x_{n}^{h}-T_{h}^{\prime}\left(x_{n}^{h}\right)^{-1} T_{h}\left(x_{n}^{h}\right), \quad n=0,1,2 \ldots
$$

To achieve this, we define the discretization on $x$ by the bounded nonlinear operators $P_{h}: X \rightarrow X_{h}$.

It has been ubserved in many computations that for sufficiently small $\mathrm{h}$ there is at most a difference of one between the number of steps required by the iterations (2) and (4) to converge to within a given tolerance $\varepsilon=0$. This is one aspect of the mesh-independence principle for Newton's method. Here we actually show that the number of steps in both iterations is the same.

The above results have already been proved in [3] but for 1 inear projections from $X$ to $X_{h}$. For special classes of boundary value problems for mesh-independence principle was proved in [1], [5]. It was used to construct certain mesh-refinement strateqies.

Wost of our resuits carry over inmediately to Newton like methoas

\section{PRELIMIKARIES}

The norms in all spaces will be denoted by the same symbol il \|

Let $F: X X \rightarrow X$ be a nonl inear operator with Lipschitz continuous Fréchet derivative on the open domain, that is

$$
\left\|F^{\prime}(x)-F^{\prime}(y)\right\| \leqslant \gamma\|x-y\|, x, y \in D, \gamma>0 .
$$

Let us assume that (1) has a simple solution and set $d=\left\|F^{\prime}\left(z^{*}\right)^{-1}\right\|$.

We will need the following theorem [8]. 
Theorem 1. Let $F: \mathbb{X}+X$ satisfy the stated hypotheses and with

$$
r^{\star}=\frac{2}{3} d_{r} \vec{r},
$$
Suppose that $U=U\left(z^{\star}, r^{\star}\right)=\left\{z \in X\|\| z-z^{\star} \|<r^{\star}\right\}$. Then for any $z_{0} \in$
$U$, iteration (2) converges to $z^{\star}$ and the iterates satisfy

$$
\left\|z_{n+1}-z^{\star}\right\| \leqslant \frac{d y\left\|z_{n}-z^{*}\right\|^{2}}{2\left(1-d y\left\|z_{n}-z^{*}\right\|\right)}, \quad n=0,1,2, \ldots
$$

Consider the family

$$
\left\{T_{h}, P_{h}\right\}, \quad h>0
$$

where

$$
T_{h}: \mathrm{D}_{h} \mathrm{CH}_{h} \rightarrow \mathrm{X}_{h}, \quad h>0
$$

are nonlinear operators and

$$
P_{h}: x \rightarrow x_{h}, h>0
$$

are nonlinear operators which are twice continuously Fréchet - differentiable at least in some bal1 U(z*, N) for some $\rho>0$ and $P_{h}(U) \subset D_{h}$.

The discretization (8) is called Lipschitz uniform if there exist scalars $\rho>0, L>0$ such that

$$
\bar{U}\left(P_{h}\left(z^{*}\right), 0\right)=D_{h}, h>0
$$

and

$$
\left\|T_{h}(w)-T_{h}^{\prime}(v) ! \leqslant L\right\| w-v \|, h>0, w, v \in \bar{U}\left(P_{h}\left(z^{\star}\right), \rho\right)
$$

Moreuver, the family (8) is called "bounded" if there exist constants $q_{1}, q_{2}>0$ such that

$$
\left\|P_{h}(u)-P_{h}\left(z^{\star}\right)\right\| \leqslant q_{1}\left\|u-z^{\star}\right\|+q_{2}\left\|u-z^{\star}\right\| \|^{2}, u \in u
$$

The motivation for the above condition is due to the identity

$$
P_{h}(u)=P_{h}\left(z^{\star}\right)+P_{h}^{\prime}\left(z^{\star}\right)\left(u-z^{\star}\right)+\frac{1}{2} P_{h}^{\prime \prime}\left(\bar{z}^{\star}\right)\left(u-z^{\star}\right)^{2},
$$

where $P_{h}^{\prime}, P_{h}^{\prime \prime}$ represent the first and second Fréchet derivat es of $P_{h}$ and $\stackrel{*}{*} \in$ i. 
Note that the identity (12) is not true, in general, for any nor.1 inear operator $P_{h}$ that is twice Fréchet-differentiable on $x$. However, it is certainly true for $P_{h}$ being a polynomial operator [4], [7].

From now on we restrict ourselves only to that subclass of twice Fréchet-differentiable nonlinear operators on $X$ that can satisfy the identity $(12)$.

Our proofs will indicate that the same results can be obtained for any nonlinear sufficiently Fréchet-differentiable operator $P_{h}$, under similar assumptions. However, due to clarity and to the fact that the main results obtained here can be achieved using the above mentioned subclass of nonlinear operators $P_{h}$, we do not pursue this goal here.

We will use the following well known estimate [7]:

$$
\left\|P_{h}(u)-P_{h}\left(z^{\star}\right)-P_{h}^{\prime}\left(z^{*}\right)\left(u-z^{\star}\right)\right\| \leqslant q_{2}\left\|u-z^{\star}\right\|^{2}
$$

Let us assume from now on that: The operators $\mathrm{Ph}_{\mathrm{h}}$ are continuously Fréchet differentiable at a closed ball $U^{\star}$ such that

$$
0 \in U^{\star} \subset U
$$

and

$$
P_{h}\left(F\left(z^{*}\right)\right)=P_{h}(0)=0, h>0
$$

The family (8) is called stable if there is $\sigma>0$ such that

$$
\left\|T_{h}^{\prime}\left(P_{h}(u)\right)^{-1}\right\| \leqslant a, u \in U, h>0
$$

and consistent of order $p$ if there are two constants $c_{0}, c_{1}>0$ such that

$$
\left\|P_{h}(F(z))-T_{h}\left(P_{h}(z)\right)\right\| \leqslant c_{0} h^{p}, z \in U, h>0
$$

and

$$
\left\|P_{h}\left(F^{\prime}(u)\right)(v)-T_{h}^{\prime}\left(P_{h}(u)\right) P_{h}(v)\right\| \leqslant c_{1} h^{P}, u, v 6 u, h>0
$$

\section{MAIN RESULTS}

We will need the following definition.

Definition $f_{2}$ by:

Let us define the numbers $r, c$ and the real functions $f_{1}$ and

$$
r=\left\|z_{0}-z^{\star}\right\|
$$




$$
\begin{aligned}
& c=\max \left(c_{0}, c_{1}\right) \\
& f_{1}(r)=3 L q_{2}\left(j r^{2}+3 L q_{1} \sigma r+16 L \sigma^{2} c \cdot h^{p}-2\right. \\
& f_{2}(r)=q_{2} r^{2}+q_{1} r+4 \sigma c h^{p}-\rho
\end{aligned}
$$

Let, $h_{1}, r_{1}$ be such that:

$$
\begin{aligned}
& h_{1}=\min \left(\frac{r^{-}}{4 \sigma c}, \frac{1}{8 L_{0}^{*} \frac{c}{c}}\right)^{\frac{1}{p}} \\
& r_{1}=\min \left(r_{1}^{+}, r_{2}^{+}, r^{\star}, \rho\right)
\end{aligned}
$$

where, $r_{1}^{+}, r_{2}^{+}$are the positive solutions of $(20)$ and (21) respectively, provided that :

$$
h \in\left[0, h_{1}\right)
$$

It is easy to check that if

$$
r \in\left[0, r_{1}\right)
$$

then

$$
f_{1}(r) \therefore 0
$$

and

$$
f_{2}(r)<0
$$

Define the real function $f_{3}$ and the number b by

$$
\begin{aligned}
& t_{3}(s)=A s^{2}+B s+C \\
& b=\left\|z^{\star}\right\|
\end{aligned}
$$

where,

$$
\begin{aligned}
& A=\frac{3}{2} L 0 \\
& B=B(r)=B_{3} r^{3}+B_{2} r^{2}+B_{1} r+B_{0} \\
& C=C(r)=C_{2} r^{2}+C_{1} r+C_{0} \\
& B_{3}=16 L \sigma q_{2} \\
& B_{2}=L o\left(4 q_{1}+8 b q_{2}-6 q_{2}\right) \\
& B_{1}=2 \operatorname{Loq}_{1} b+2 L o q_{2} b^{2}-4 q_{1}-4 b q_{2} \\
& B_{0}=-\left(\operatorname{Loq}_{1} b+\operatorname{Lq}_{2} 0+1\right)
\end{aligned}
$$


and

$$
\begin{aligned}
& c_{2}=6 q_{2} \\
& c_{1}=4\left(q_{1}+b q_{2}\right) \\
& c_{0}=2 c h^{p}+q_{1} b+q_{2} b^{2}
\end{aligned}
$$

We now observe the following:

(a) $\mathrm{B}_{0}<0, \quad \mathrm{~B}_{3}>0$

(b) The function $B^{2}-4 A C$ is a sixth degree polynomial with the coefficient of the highest power being positive and the constant given by

$$
B_{0}^{2} \quad 4 A C_{0}=E_{1} q_{1}^{2}+E_{2} q_{2}^{2}+E_{3} q_{1} q_{2}+E_{4} q_{1}+E_{5} q_{2}+E_{6}
$$

where,

$$
\begin{aligned}
& E_{1}=(L \sigma b)^{2} \\
& E_{2}=(L \sigma)^{2} \\
& E_{3}=2(L \sigma)^{2} b \\
& E_{4}=-4 L \sigma b \\
& E_{5}=2 L \sigma\left(1-4 b^{2}\right) \\
& E_{6}=1-12 L c \sigma^{2} h^{p} \\
& (c) \text { Define the function g by } \\
& g(k)=A\left(4 A B^{2}-C^{\prime}\right) k^{2}+4 A B \sigma c k+(\sigma c)^{2}, C^{\prime}=C-2 \sigma c^{p}
\end{aligned}
$$

The function $4 A B^{2}-C^{\prime}$ is a sixth degree polynomial with the coefficient of the highest power being positive and the constant given by

$$
4 A B_{0}^{2}-q_{1} b-q_{2} b^{2}=E_{1}^{1} q_{1}^{2}+E_{2}^{1} q_{2}^{2}+E_{3}^{1} q_{1} q_{2}+E_{4}^{1} q_{1}+E_{5} q_{2}^{1}+E_{6}^{1}
$$

where,

$$
\begin{aligned}
& E_{1}^{1}=6(L \sigma)^{3} b^{2} \\
& E_{2}^{1}=6(L \sigma)^{3} \\
& E_{3}^{1}=12(L \sigma)^{3} b
\end{aligned}
$$




$$
\begin{aligned}
E_{q}^{1} & =\left[12(L \sigma)^{2}-1\right] b \\
E_{5} & =12(L \sigma)^{2}-b^{2} \\
E_{6} & =6 L \sigma \\
\text { Let } q_{1}^{*} & =\max \left(-\frac{E_{4}}{E_{1}},-\frac{E_{4}^{1}}{E_{1}^{1}}\right), q_{2}^{*}=\max \left(-\frac{E_{5}}{E_{2}},-\frac{E_{5}^{1}}{E_{2}^{1}}\right)
\end{aligned}
$$

and

$$
k^{*}=\operatorname{nax}\left(-\frac{O C}{2 A B}, k_{2}\right)
$$

where $k_{\ell}$ is the large solution of the equation

$$
g(k)=0
$$

Choose $q_{1}, q_{2}, k, h$ and $h_{2}$ such that:

$$
\begin{aligned}
& q_{1} \geqslant q_{1}^{*} \\
& q_{2} \geqslant q^{*} \\
& k \geqslant k^{*}
\end{aligned}
$$

and

$$
h<h_{2}=\min \left(\left(\frac{1}{12 L c \sigma^{2}}\right)^{\frac{1}{p}},\left(\frac{1}{L k \sigma}\right)^{\frac{1}{p}}, h_{1}\right)
$$

It is easy to check that we can find $r_{2}>0$ such that if $r \in\left[0, r_{3}\right)$ where

$$
r_{3}=\min \left(r_{1}, r_{2}\right)
$$

then the following are true:

$$
\begin{aligned}
& B<0 \\
& B^{2}-4 A C \geqslant 0 \\
& \sigma C+2 A k B \leqslant 0 \\
& g(k) \geqslant 0
\end{aligned}
$$

and

$$
0<s \leqslant k h^{p}<\frac{1}{L \sigma}
$$

where $s$ is the small solution of the equation

$$
f_{3}(s)=0
$$


that

$$
\text { Let } \varepsilon>0 \text {, since } z_{n} \rightarrow z^{\star} \text { as } n \rightarrow \infty \text { there exists } N=N(\varepsilon) \text { such }
$$

$$
\left\|z_{n}-z^{*}\right\| \leqslant\left[\frac{1}{q_{2}} k_{1} h^{p}\right]^{\frac{1}{2}} \text { for some } k_{1}>0
$$

Finally, define the numbers $k_{2}, B, r_{4}, h_{4}$ and the function $f_{4}$ by

$$
k_{2}=k+2 \sigma c+k_{1}
$$

$B=\min \left(2\left[\bar{q}_{1}-\left(q_{1}+q_{2}\right)\right], \frac{1}{q_{1}+q_{2}}\right)$ for some fixed number $\bar{q}_{1}$ with

$$
\begin{gathered}
\bar{q}_{1}>q_{1}+q_{2} \\
h_{3}=\min \left\{h_{2},\left(\frac{\beta}{(1+2 \beta) L o^{2} c}\right)^{\frac{1}{p}},\left(\frac{\left(q_{1}+q_{2}\right)}{k_{2}}\right)^{\frac{1}{p}}\right\} \\
f_{4}(r)=\operatorname{Loq}_{2}(1+\beta) r^{2}+\operatorname{Loq}_{1}(1+\beta) r+(1+2 \beta) h^{p}-\beta
\end{gathered}
$$

and $r_{4}$ to be the positive solution of the equation

$$
f_{4}(r)=0
$$

guaranteed to exist by the choice of $h_{3}$ and $B$

Let $h, r$ be such that:

$$
h \in\left(0, h_{3}\right)
$$

and

$$
r \in\left[0, r_{4}\right)
$$

then it can easily be checked that

$$
f_{4}(r) \leqslant 0
$$

We can now prove the first result.

\section{Theorem 2}

Let $F: \mathbb{X} \rightarrow X$ be a nonlinear operator satisfying the hypotheses of theorem 1 and consider a Lipschitz uniform discretization (8) which is "bounded", stable and consistent of order $p$.

Assume:

$$
\begin{aligned}
& q_{1} \geqslant q_{1} \\
& q_{2} \geqslant q_{2} \\
& k \geqslant k^{\star}
\end{aligned}
$$


and that (14) and (15) are satisfied.

Then equation (3) has a locally unique solution $x_{h}^{\star}$ such that:

$$
x_{h}^{\star}=P_{h}\left(z^{*}\right)+O\left(h^{p}\right)
$$

for all $h \in\left(0, h_{1}\right]$.

There exist constants $h \in\left(0, h_{2}\right]$ and $r \in\left(0, r_{3}\right)$ such that the iteration (4) converges to $x_{h}^{\star}$.

Moreover, the following are true:

$$
\begin{aligned}
& x_{n}^{h}=P_{h}\left(z_{n}\right)+o\left(h^{p}\right) \\
& T_{h}\left(x_{n}^{h}\right)=P_{h}\left(F\left(z_{n}\right)\right)+o\left(h^{p}\right)
\end{aligned}
$$

and

$$
x_{n}^{h}-x_{h}^{\star}=P_{h}\left(z_{n}-z^{\star}\right)+O\left(h^{p}\right)
$$

for $n=0,1,2 \ldots$ and all $z_{0} \in U\left(z^{\star}, r_{3}\right)$.

Proof. Using the Newton-Kantorovich theorem [7], (15) we show exactly as in [3] that (3) has a solution $x_{h}^{*} \in \bar{U}\left(p_{h}\left(z^{*}\right), r(h)\right)$ which is unique in $U\left(P_{h}\left(z^{\star}\right), \bar{r}(h)\right)$ where

$$
\begin{aligned}
& \alpha(h)=2 \sigma L\left\|T_{h}\left(P_{h}\left(z^{*}\right)\right)^{-1} T_{h}\left(P_{h}\left(z^{*}\right)\right)\right\| \\
& r(h)=\frac{1}{\sigma L}(1-\sqrt{1-\alpha(h)}) \leqslant \rho \\
& \bar{r}(h)=\frac{1}{\sigma L}(1+\sqrt{1-\alpha(h)})
\end{aligned}
$$

Moreover,

$$
\left\|x_{h}^{\star}-P_{h}\left(z^{*}\right)\right\| \leqslant r(h) \leqslant 20 c_{0} h^{p}
$$

which proves (53). to $x_{h}^{*}$ if

As in [3] by applying theorem 1 to (3) we see that (4) converges

$$
\left\|P_{h}\left(z_{0}\right)-x_{h}^{*}\right\|<\frac{2}{3\left\|T_{h}^{\prime}\left(x_{h}^{*}\right)^{-1}\right\|}
$$

and

$$
\bar{U}\left(x_{h}^{\star},\left\|P_{h}\left(z_{0}\right)-x_{h}^{\star}\right\|\right) \subset \bar{U}\left(P_{h}\left(z^{\star}\right), \rho\right)
$$

that is if 


$$
q_{2}\left\|z_{0}-z^{\star}\right\|^{2}+q_{1}\left\|z_{0}-z *\right\|+4 a c_{0} h^{p}<\frac{2-4 \sigma^{2} L c_{0} h^{p}}{3 L \sigma}
$$

and

$$
q_{2}\left\|z_{0}-z^{\star}\right\|^{2}+q_{1}\left\|z_{0}-z^{\star}\right\|+4 o c_{0} h^{p} \leqslant \rho
$$

hold respectively.

But (63) and (64) can now be written as

$$
f_{1}(r)=0
$$

and

$$
f_{2}(r) \leqslant 0
$$

which are true by the choice of $h, r, h_{1}$ and $r_{1}$. $z_{0} \in u\left(z^{*}, r_{3}\right)$

We now show, using induction as in [3] that for $h \in\left(0, h_{3}\right)$,

$$
\| x_{n}^{h}-p_{h}\left(z_{n}\right) ! \leqslant s, \quad n=0,1,2, \ldots
$$

By rearranging the sequences in (65) we can start the induction from $n=0$ if necessary. The above inequal ity is true for $n=0$. Let us assume that it is true for $n=0,1,2, \ldots, i$.

\section{Then}

$$
\begin{aligned}
& x_{i+1}^{h}-P_{h}\left(z_{i+1}\right)=T_{h}^{\prime}\left(x_{j}^{h}\right)^{-1}\left\{\left[T_{h}^{\prime}\left(x_{i}^{h}\right)\left(x_{i}^{h}-P_{h}\left(z_{i}\right)\right)-T_{h}\left(x_{i}^{h}\right)+T_{h}\left(P_{h}\left(z_{i}\right)\right]\right.\right. \\
& +\left[\left(T_{h}^{\prime}\left(x_{i}^{h}\right)-T_{h}^{\prime}\left(P_{h}\left(z_{j}\right)\right)\right) P_{h}\left(F^{\prime}\left(z_{i}\right)^{-1}\left(z_{j}\right)\right)\right]+\left[T _ { h } ^ { \prime } \left(P _ { h } ( z _ { i } ) P _ { h } \left(F^{\prime}\left(z_{i}\right)^{-1} F\left(z_{j}\right)\right.\right.\right. \\
& \left.\left.-D_{i}\left(F\left(z_{j}\right)\right)\right]+\left[P_{h}\left(F\left(z_{i}\right)\right)-T_{h}\left(P_{h}\left(z_{i}\right)\right)\right]\right\}+\left[P_{h}\left(z^{*}\right)-P_{h}\left(z_{i}\right)+P_{h}^{\prime}\left(z^{*}\right)\left(z_{i}-z^{*}\right)\right] \\
& -P_{h}^{\prime}\left(z^{*}\right)\left(F^{\prime}\left(z_{j}\right)^{-1} F\left(z_{j}\right)+P_{h}^{\prime \prime}\left(z^{*}\right)\left(z_{i+1}-z^{*}\right)^{2}+P_{h^{\prime}}\left(F^{\prime}\left(z_{j}\right)^{-1} F\left(z_{j}\right)\right)\right.
\end{aligned}
$$

We nuis have, using (10), (13).

$$
\begin{aligned}
& \left\|P_{h}\left(z^{\star}\right)-P_{h}\left(z_{j}\right)+P_{h}^{\prime}\left(z^{\star}\right)\left(z_{i}-z^{\star}\right)\right\| \leqslant q_{2}\left\|z_{i}-z^{\star}\right\|^{2} \\
& \left\|P_{h}^{\prime}\left(z^{\star}\right)\left(z_{i}-z_{i+1}\right)\right\| \leqslant q_{1}\left\|z_{i}-z_{i+1}\right\|
\end{aligned}
$$




$$
\begin{aligned}
& \left\|P_{h}^{\prime \prime}\left(z^{\star}\right)\left(z_{i+1}-z^{*}\right)^{2}\right\| \leqslant q_{2}\left\|z_{i+1}-z^{*}\right\|^{2} \\
& \left\|P_{h}\left(z_{i}-z_{i+1}\right)\right\| \leqslant\left\|P_{h}\left(z^{\star}\right)\right\|+q_{1}\left\|z_{i}-z_{i+1}-z^{*}\right\|+ \\
& +q_{2}\left\|z_{i}-z_{i+1}-z^{\star}\right\|^{2}
\end{aligned}
$$

By theorem 1, $\left\|z_{i+1}-z^{\star}\right\| \leqslant\left\|z_{i}-z^{*}\right\| \leqslant \ldots \leqslant\left\|z_{0}-z^{\star}\right\|$ and the result on the norm of the braces above in [3] we have that the right hand side of (66) is bounded by

$$
\begin{aligned}
& \frac{\sigma}{1-\operatorname{Los}}\left[\frac{1}{2} L s^{2}+2 L\left(q_{1}(2 r+b)+q_{2}(2 r+b)^{2}\right) r s+2 c h^{P}\right] \\
& +q_{2} r^{2}+2 q_{1} r+q_{2} r^{2}+2 q_{1} r+q_{1} b+4 q_{2} r^{2}+q_{2} b^{2}+4 b q_{2} r=s
\end{aligned}
$$

That is,

$$
\left\|x_{h}^{h}-P\left(z_{h}\right)\right\| \leqslant s \leqslant k h^{p}
$$

which proves (54).

Equation (55) can be proved exactly as in [3].

Finally, for some $\bar{z} \in U^{*}$

$$
\begin{aligned}
& \left\|\left(x_{n}^{h}-x_{h}^{\star}\right)-P_{h}\left(z_{n}-z^{\star}\right)\right\| \leqslant\left\|x_{n}^{h}-P_{h}\left(z_{n}\right)\right\|+\left\|x_{h}^{\star}-P_{h}\left(z^{\star}\right)\right\| \\
& +\left\|\left[P_{h}(0)+\left(P_{h}\left(z^{\star}\right)-P_{h}\left(z_{n}\right)+P_{h}^{\prime}(\bar{z})\left(z_{n}-z^{\star}\right)\right)\right]\right\| \\
& \leqslant k h^{p}+20 c h^{p}+q_{2}\left\|z_{n}-z^{\star}\right\|^{2} \leqslant k_{2} h^{p}
\end{aligned}
$$

by (13), (14), (15), (44), (45), (66), (67) and (68) which proves (56) and completes the proof of the theorem.

We now prove the last result.

\section{Theorem 3}

Assume:

(1) The hypotheses of theorem 2 hold;

(2) There exist constants $\bar{q}_{1}, \bar{q}_{2}$ such that

$\lim _{n>0} \inf \left\|p_{h}(z)\right\| \geqslant \bar{q}_{1}\|z\|+\bar{q}_{2}\|z\|^{2}$ for $z \in \bar{U}$ and $\bar{q}_{1}>q_{1}+q_{2}$ 
Then for $r \in\left(0, r_{4}\right)$, and for any fixed $0<\epsilon \leqslant 1$ and $z_{0} \in \bar{U}\left(z^{\star}, r\right)$ there exists $h_{4}=h_{4}\left(6, z_{0}\right) \in\left(0, h_{6}\right)$ where,

$$
h_{6}=\min \left(h_{3}, h_{5}\right)
$$

and $h_{5}=h\left(z_{0}\right)$ is such that

$$
\left\|P_{h}\left(z_{i}-z^{*}\right)\right\| \geqslant \vec{q}_{1}\left\|z_{i}-z^{\star}\right\|+\bar{q}_{2}\left\|z_{i}-z^{*}\right\|^{2} h \in\left(0, h_{5}\right)
$$

and $i$ is the unique integer defined by

$$
\left\|z_{i+1}-z^{\star}\right\|<\epsilon \leqslant\left\|z_{i}-z^{\star}\right\|
$$

such that

$$
\left|\min \left\{n \geqslant 0,\left\|z_{n}-z^{\star}\right\|<\epsilon\right\}-\min \left\{n \geqslant 0,\left\|x_{n}^{h}-x_{h}^{\star}\right\|<\epsilon\right\}\right| \leqslant 1
$$

for $a_{11} h \in\left(0, h_{4}\right]$.

Proof. By (68) and the choice of $h, \beta$ we have

$$
\begin{gathered}
\left\|x_{i+1}^{h}-x_{h}^{*}\right\| \leqslant\left\|p_{h}\left(z_{i+1}-z^{*}\right)\right\|+k_{2} h^{p} \leqslant q_{1}\left\|z_{i+1}-z^{*}\right\|+q_{2}\left\|z_{i+1}-z^{*}\right\|^{2} \\
+k_{2} h^{p} \leqslant\left(q_{1}+q_{2}\right) \epsilon+k_{2} h^{p}<2\left(q_{1}+q_{2}\right) \epsilon
\end{gathered}
$$

Using theorem 1 , and the choice of $h, \beta, r$ we get

$$
\begin{aligned}
\left\|x_{i+2}^{h}-x_{h}^{\star}\right\| & \leqslant \frac{L \sigma\left\|x_{i+1}^{h}-x_{h}^{\star}\right\|^{2}}{2\left(1-L \sigma\left\|x_{i+1}^{h}-x_{h}^{\star}\right\|\right)} \\
& \leqslant \frac{L \sigma\left\|x_{0}^{h}-P_{h}\left(z^{\star}\right)+P_{h}\left(z^{\star}\right)-x_{h}^{\star}\right\|}{2\left(1-L \sigma\left\|x_{0}^{h}-P_{h}\left(z^{\star}\right)+P_{h}\left(z^{\star}\right)-x_{h}^{\star}\right\|\right)}\left\|x_{i+1}-x_{h}^{\star}\right\| \\
& <\frac{L \sigma\left[q_{1}\left\|z_{0}-z^{\star}\right\|+q_{2}\left\|z_{0}-z^{\star}\right\|^{2}+2 \sigma c h^{P}\right]}{\left[1-L \sigma\left(q_{1}\left\|z_{0}-z^{\star}\right\|+q_{2}\left\|z_{0}-z^{\star}\right\|^{2}+2 \sigma c h^{P}\right)\right]}\left(q_{1}+q_{2}\right) \epsilon \\
& \leqslant B\left(q_{1}+q_{2}\right) \epsilon<\epsilon
\end{aligned}
$$

Moreover, by (68), (69) and (70),

$$
\begin{aligned}
& \bar{q}_{1} \epsilon+\bar{q}_{2} \epsilon^{2} \leqslant q_{1}\left\|z_{i}-z^{\star}\right\|+q_{2}\left\|z_{i}-z^{\star}\right\|^{2} \\
& \leqslant\left\|P_{h}\left(z_{i}-z^{\star}\right)\right\| \leqslant\left(\left\|x_{i}^{h}-x_{h}^{\star}\right\|+k_{2} h^{p}\right)
\end{aligned}
$$


or

$$
\left\|x_{i}^{h}-x_{h}^{*}\right\| \geqslant \bar{q}_{1} \epsilon+\bar{q}_{2} \epsilon^{2}-c_{2} h^{p}
$$

If $\left\|x_{i-1}^{h}-x_{h}^{*}\right\|<\epsilon$ then as in (74) we get

$$
\left\|x_{i}^{h}-x_{h}^{*}\right\|<\frac{1}{2} \beta G
$$

By, (75) and (76) we must also have

$$
\vec{q}_{1} \epsilon+\bar{q}_{2} \epsilon^{2}-k_{2} h^{p}<\frac{1}{2} B \epsilon
$$

or

$$
\bar{q}_{1} \epsilon+\bar{q}_{2} \epsilon^{2}-\left(q_{1}+q_{2}\right) \epsilon<\frac{1}{2} B E
$$

or

$$
\bar{q}_{2}<\frac{1}{\epsilon}\left(\frac{1}{2} \beta+q_{1}+q_{2}-\bar{q}_{1}\right)<0 \text {, }
$$

that is

$$
\overline{\mathrm{q}}_{2}<0,
$$

which is a contradiction. Therefore, we have

$$
\left\|x_{j-1}^{h}-x_{h}^{\star}\right\| \geqslant E \text {. }
$$

The result is now obtained by (71), (74) and (77).

We can do better sometimes. Let us assume that:

$$
0<\mathrm{q}_{1}<1
$$

and

$$
\bar{q}_{1}+\bar{q}_{2}>\max \left(\frac{1}{2}, \frac{q_{1}+2 q_{2}}{4}, \frac{q_{2}}{2\left(1-q_{1}\right)}\right)
$$

Define $\beta_{1}>0$ by

$B_{1}=\min \left(\frac{1}{R}, B, 2 \bar{q}_{2}, 2\left(\bar{q}_{1}+\bar{q}_{2}\right)-1, \frac{4\left(\bar{q}_{1}+\bar{q}_{2}\right)-\left(a_{1}+2 q_{2}\right)}{2}, \frac{2\left(1-q_{1}\right)\left(\bar{q}_{1}+\bar{q}_{2}\right)-q_{2}}{1-q_{1}}\right)$

Choosing $R, h_{7}>0$ such that:

$\max \left(\frac{1}{2}, \frac{q_{1}+2 q_{2}}{4}, \frac{q_{2}}{2\left(1-q_{1}\right)}\right)<R \leqslant \bar{q}_{1}+\bar{q}_{2}-\frac{1}{2} \beta^{\star}$, for some $\beta^{\star} \in\left(0, B_{1}\right)$,

$h_{7}=\min \left(n_{6},\left(\frac{R E^{2}}{k_{2}}\right)^{\frac{1}{p}},\left(\frac{8 R-r q_{2}-q_{1}}{k_{2}}\right)^{\frac{1}{p}},\left(\frac{\left(2 R-a_{2}\right)\left(2 R-a_{2}-2 R q_{1}\right.}{4 R^{2}\left(2 R-q_{2}\right) k_{2}}\right)^{\frac{1}{p}}\right)$. 
Here, $E \in\left[E^{+}, \frac{1}{2 R}\right]$ and $E^{+}$is the positive solution of the equation

$$
\left(2 R-q_{2}\right) E^{2}-q_{1} E-k_{2} h^{p}=0,
$$

guaranteed to exist by the choice of $h$ and $R$.

$$
\begin{aligned}
& \text { Define } r_{5}(r)=\operatorname{Loq}_{2}\left(1+\beta^{\star}\right) r^{2}+\operatorname{Loq}_{1}\left(1+\beta^{\star}\right) r+\left(1+2 \beta^{\star}\right) h^{p}-\beta^{\star}=0 \text {, }
\end{aligned}
$$

guaranteed to exist by the choice of $h, R$ and $b^{*}$.

Set

$$
r_{6}=\min \left(r_{4}, r_{5}\right) \text {. }
$$

h. $R, B^{*}$ We can now casily check that by $(78),(79)$ and the choice of

$$
0 \cdot \epsilon^{+}<\frac{1}{2 \bar{R}}+.
$$

We can show exactly as in theorem 3 that:

$$
\begin{aligned}
& \left\|x_{i+1}^{h}-x_{h}^{\star}\right\| \leqslant q_{2} \epsilon^{2}+a_{1} E+k_{2} h^{P}<2 R \epsilon^{2} \\
& \left\|x_{i+2}^{h}-x_{h}^{*}\right\| \leqslant B^{\star} R \epsilon^{*}<\epsilon^{2} \\
& \left\|x_{i-1}^{h}-x_{h}^{\star}\right\| \geqslant \epsilon^{2} .
\end{aligned}
$$

Then by theorein 3

$\left|\min \left\{n \geq 0,\left\|z_{n}-z^{*}\right\|<\epsilon\right\}-\min \left\{n \geqslant 0,\left\|x_{n}^{h}-x_{n}^{*}\right\|<\epsilon^{2}-\epsilon\right\}\right| \leqslant 2$.

Mureover,

$$
\left\|x_{i+1}^{n}-x_{n}^{*}\right\| \leqslant 2 R \epsilon^{2} \leqslant \epsilon .
$$

Therefors (80), because of (81), becomes

$I=\min \left\{n \geqslant y \mid x_{n}^{h}-x_{h}^{*} \|<\epsilon\right\}-\min \left\{n \geqslant 0,\left\|z_{n}-z^{\star}\right\|<\epsilon\right\}, \quad I=-1,0$.

We have now proved the following:

Corollary. Assume that the hypotheses of theorem 3, (78) and (79) hold. Then for $r \in\left(0, r_{6}\right)$, and for any $\in \in\left[E^{+}, \frac{1}{2 R}\right]$ and $z_{0} \in \bar{U}(\bar{z}, r)$ there exists $h_{8}=h_{8}\left(\epsilon, z_{0}\right) \in\left(0, h_{7}\right)$ such that: 
$I=\min \left\{n \geqslant 0,\left\|x_{n}^{h}-x_{h}^{\star}\right\|<\epsilon\right\}-\min \left\{n \geqslant 0,\left\|z_{n}-z^{\star}\right\|<\epsilon\right\}$, with

$1=-1,0$

for all h $\in\left(0, h_{8}\right]$.

in [3].

The above result improves and extends the corresponding one Note that (82) iminties that:

$\min \left\{n \geqslant 0,\left\|x_{n}^{h}-x_{h}^{*}\right\|<E\right\} \leqslant$ inin $\left\{n \geqslant 0,\left\|z_{n}-z^{*}\right\|<\varepsilon\right\}$.

Inequality (83) arises the iuestion of the actual equality being achieved in (83). The proof of (83) was based primarily on (7), (56) and (69).

We note:

$$
\begin{aligned}
x_{n}^{h}-x_{h}^{*} & =P_{h}\left(z_{n}-z^{\star}\right)+0\left(h^{p}\right) \\
= & P_{h}(0)+P_{h}^{\prime}(\bar{z})\left(z_{n}-z^{\star}\right)+0\left(h^{p}\right) \text {, for some } \\
& \bar{z} \in U^{\star} \\
= & P_{h}^{\prime}(\bar{z})\left(z_{n}-z^{\star}\right)+O\left(h^{p}\right) .
\end{aligned}
$$

Assume that the inverse of $P_{h}^{\prime}(\bar{z})$ exists and is bqunded for all sufficiently small $h$ and $\bar{z} \in U^{*}$. Set $L_{h}=\left(P_{h}^{\prime}(\bar{z})\right)^{-1}$ and $\bar{q}_{l}^{*} \geqslant\left\|L_{h}\right\|$.

Then the equation (84) can be written as

$$
z_{n}-z^{\star}=L_{h}\left(x_{n}^{h}-x_{h}^{*}\right)+0\left(h^{p}\right), \quad n=0,1,2, \ldots, h \text { sufficiently sma } 17 \text {; }
$$$$
\begin{aligned}
& \text { Es theorem } 1 \text { we can have, } \\
& \left\|x_{n+1}^{h}-x_{h}^{\star}\right\| \leqslant \frac{L \sigma \| x_{n}^{h}-x_{h n^{*}}^{2}}{2\left(1-L \sigma\left\|x_{n}^{h}-x_{h}^{\star}\right\|\right)}, n=0,1,2, \ldots .
\end{aligned}
$$

Moreover, note that the assumption on $P_{h}$ given by (11), (13), (14), and $(15)$ hold for the Tinear operator $L_{h}$ with $q_{1}=\bar{q}_{1}^{*}, q_{2}=0$.

If the rest of the assumptions made for $P_{h}$ hold for $L_{h}$ then the proofs of theorem 2 will go through for $L_{h}$.

Finally, assume that:

$\lim _{h \rightarrow 0} \inf \left\|L_{h}(x)\right\| \geqslant \bar{q}_{1}^{*}\left\|x-x_{h}^{*}\right\|+\bar{q}_{2}^{*}\left\|x-x_{h}^{*}\right\|^{2}, x \in \bar{U}\left(P_{h}\left({ }^{*}\right), b\right)$ $\left(\bar{a}_{2}^{*}=0\right)$. 
If we now interchange the role of the $z^{\prime} s$ with the $x^{\prime} s$ in theorems $1,2,3$ and the Corollary, under similar assumptions on $h, \rho_{h}^{\star}=\left\|x_{0}^{h}-x_{h}^{*}\right\|, \bar{q}_{1}^{\star}, \bar{q}_{2}^{\star}, q_{1}^{\star}$ the proofs of both theorem 3 and the corollary will go through. Therefore, we can show that:

$$
\min \left\{n \geqslant 0,\left\|x_{n}^{h}-x_{h}^{\star}\right\|<\epsilon\right\} \geqslant \min \left\{n \geqslant 0,\left\|z_{n}-z^{\star}\right\|<\epsilon\right\},
$$

for sufficiently small $h$ and $\rho_{h}^{*}$.

By (83) and (88) we finally obtain

$\min \left\{n \geqslant 0,\left\|x_{n}^{h}-x_{h}^{\star}\right\|<\epsilon\right\}=\min \left\{n \geqslant 0,\left\|z_{n}-z^{\star}\right\|<\epsilon\right\}$,

where $h \in\left(0, h_{9}\right], z_{0} \in \bar{U}\left(z^{\star}, r\right), x_{0}^{h} \in \bar{U}\left(P_{h}\left(z^{\star}\right), \rho\right)$ and any $\epsilon \in\left[\epsilon_{1}^{+}, \frac{1}{2 R_{1}}\right] \cap\left[\epsilon_{1}, \frac{1}{2 R}\right]$ provided that the intersection is nonempty. Here, $h_{9}=\min \left(h_{8}, h_{8}^{\star}\right), r \in\left(0, r_{6}\right), \rho \in\left(0, \rho_{6}\right)$. Finally,
the $h_{8}^{\star}, \rho_{6}, \rho_{1}^{+}$and $R_{1}$ were found as the $h_{8}, r_{6}, \epsilon^{+}$and $R$ respectively. The result given by equation (89) improves and extends the corresponding result in [3].

\section{REMARKS - EXAMPLES}

The condition (15) does not hold in general for any nonl inear projections $P_{h}, h>0$. However, it holds for linear projections [3] and in fact for multilinear projections [4].

In particular, it has been shown in [4] that when $F$ is a quadratic operator and the $P_{h}$ are quadratic projections on $\mathbb{R}^{n}$ (or $\mathbb{Q}^{n}$ ) then (3) can be replaced with a quadratic system on $\mathbb{R}^{n}$ (or $\mathbb{C}^{n}$ ). 


\section{REFERENCIAS}

[1] ALLGOWER, E.L. and MCCORMICK, S.F. Newton's method with mesh refine ments for numerical solution of nonlinear two-point boundary value problems. Numer. Math. 29 (1978), 237-260.

[2] ALLGOWER, E.L., MCCORMICK, S.F. and PRYOR, D.V. A general mesh independence principle for Newton's method applied to second order boundary value problems. Computing 23 (1979), 233-246.

[3] ALLGOWER, E.L., BUHMER, K., POTRA, F.A. and RHEINBOLDT, W.C. A meshindependence principle for operator equations and their discretiza tions. SIAM J. Num. Anal. Vol. 23, 1, (1986), 160-169.

[4] ARGYROS, I.K. Quadratic equations and applications to Chandrasekhar's and related equations. Bull. Austral. Math. Soc. Vol. 32 (1985), 275292.

[5] MCCORMICK, S.F. A revised mesh reforcement strategy for Newton's method applied to nonlinear two-point boundary value problems. Lecture Notes in Mathematics 679, Springer-Verlag Pub1. 1978, 15-23.

[6] ORTEGA, J.M. and RHEINBOLDT, W.C. On discretization and differentiation of operators with application to Newton's method. SIAM J. Num. Anal. Vol. 3, (1966), 143-156.

[7] Iterative solutions of nonlinear equations in several variables. Academic Press, New York, 1970.

[8] RHEINBOLDT, W.C. An adaptive continuation process for solving systems of nonlinear equations. Polish Academy of Science. Banach Ctr. Publ. $3(1977), 129-142$.

Ioannis K. Argyros

Department of Mathematics

New Mexico State University

Las Cruces, NM 88003 . 